\title{
Muscle physiology: move to translation
}

\author{
Coen A. C. Ottenheijm • Richard T. Jaspers • \\ Rob C. I. Wüst · Jolanda van der Velden
}

Published online: 18 March 2014

(c) Springer International Publishing Switzerland 2014

Since the discovery of the striation pattern of muscle by Antoni van Leeuwenhoek in the Netherlands, research in muscle physiology has provided an immensely detailed picture of skeletal and cardiac muscle structure and function. Particularly in recent years, molecular techniques, gene expression and imaging techniques have provided a wealth of information about the basic function of muscle and its dysfunction in chronic diseases and congenital myopathies. In this special issue, based on the work presented at the 42nd European Muscle Conference (2013) held in Amsterdam (The Netherlands), an overview of the state-of-the-art in skeletal and cardiac muscle research is presented. Since basic and clinical muscle physiology are two sides of the same coin, combining both perspectives is key to fully profit from advances made in both research fields.

The force-generating capacity of muscle is largely determined by the quantity and quality of the contractile apparatus. As striated muscle is generally considered as post-mitotic tissue, hypertrophy relies on a net positive balance between the rate of protein synthesis and degradation. In skeletal muscle including the diaphragm, muscle stem cells, residing between the myofiber basal lamina and sarcolemma (satellite cell, MuSC) have the ability to

C. A. C. Ottenheijm · R. C. I. Wüst · J. van der Velden $(\square)$ Department of Physiology, Institute for Cardiovascular Research (ICaR-VU), VU University Medical Center, van der

Boechorststraat 7, 1081 BT Amsterdam, The Netherlands e-mail: j.vandervelden@vumc.nl

\section{R. T. Jaspers}

Laboratory for Myology, MOVE Research Institute Amsterdam, Faculty of Human Movement Sciences, VU University Amsterdam, van der Boechorststraat 9, 1081 BT Amsterdam, The Netherlands proliferate, differentiate and fuse with the host myofiber. Whether the pool of myonuclei (i.e. amount of DNA) within a myofiber is limiting protein synthesis, and as such myofibrillar hypertrophy, is still subject of debate. In this issue, Blaauw and Reggiani (2014) discuss the current theories on the role of MuSC in muscle hypertrophy. Is the activation of MuSC and accretion of myonuclei within the myofiber requisite for its hypertrophy? Recent reports suggest that MuSC activation may not be required. Blaauw and Reggiani (2014) argue that local inflammatory responses (elevated cytokines) may attract and activate other muscle resident stem cells, such as mesangioblasts which can differentiate into muscle precursors and possibly contribute to the pool of myonuclei within the myofiber.

A key alternative mechanism to increase the rate of protein synthesis is the activation of the mammalian target of rapamycin (mTOR), which is activated by mechanical loading, leucine or growth factors in muscle. Activation of mTOR is generally regarded as a prime stimulator of mRNA translation. In this issue of the journal, Jacobs et al. (2013) reviewed the current literature on how mechanical and biochemical stimuli activate mTOR signaling. Recent evidence from Hornberger's laboratory suggests that the late endosomal lysosome could serve as a prime regulatory center for controlling the mechanical loading induced activation of mTOR in skeletal muscle. These novel insights suggest that the late endosome/lysosome system plays a critical role in protein synthesis, protein trafficking as well as protein degradation.

In addition to muscle fiber size, also muscle quality matters. Both skeletal and cardiac muscle function can be negatively affected by lifestyle and ageing, particularly through an increased oxidative stress. Canton et al. (2014) discuss the role of secondary mechanisms, particularly mediated by oxidative stress, in muscular dystrophies, 
primary caused by gene mutations. In addition to the inherited defect, secondary modifiers contribute to the progression of muscle wasting and weakness. Likewise, secondary disease-mechanisms are important contributors to the progression of heart failure.

In addition to post-translation modifications mediated by oxidative stress, genetic mutations play a key role in the pathophysiology of cardiac dysfunction. Recent advances in genetics have suggested that disease-causing mutations are present in more than half of patients with hypertrophic cardiomyopathy and idiopathic dilated cardiomyopathy. These inherited cardiomyopathies, frequently caused by mutations in genes encoding sarcomeric proteins, are a major cause of morbidity and mortality. In this issue, Poggesi and Ho (2014) describe the characteristics of hypertrophic cardiomyopathy and stress the importance of studies on the mechanisms which are induced by sarcomere mutations and which may be target for future therapeutic interventions. Due to major advances in proteomic analyses of post-translational protein modifications, cellular components have been identified which may be druggable targets for treatments to prevent and/or delay congenital muscle disease.

In chronic heart failure, myocardial efficiency (cardiac work divided by oxygen consumption) is considerably diminished (Wong et al. 2010), which may be due to altered catecholamine handling. van Eif et al. (2013) tested the possibility that oxidation of catecholamines in failing hearts is induced by increased monoamine oxidase A (MAO-A) activity within cardiac myocytes and relate to increased number of intrinsic cardiac adrenergic (ICA) cells. Indeed, rat right-ventricular overload was accompanied by elevated MAO-A activity, but occurred without an increase number of ICA cells. The possibility is discussed that ICA cell activity is increased in the failing heart rather than its density. The role of MAO-A and catecholamine oxidation in the transition from myocardial hypertrophy to chronic heart failure warrants further investigation.

Despite major recent advances made in muscle research, some of which are highlighted in this issue of the Journal of Muscle Research and Cell Motility, the causes of many myopathies remain unresolved and successful treatment strategies are scarce. To establish novel research strategies to unravel muscle disease initiation and progress, research groups need to join forces and bridge the gap between preclinical and clinical departments.

\section{References}

Blaauw B, Reggiani C (2014) The role of satellite cells in muscle hypertrophy. J Muscle Res Cell Motil. doi:10.1007/s10974-0149376-y

Canton M, Menazza S, di Lisa F (2014) Oxidative stress in muscular dystrophy: from generic evidence to specific sources and targets. doi:10.1007/s10974-014-9380-2

Jacobs BL, Goodman CA, Hornberger TA (2013) The mechanical activation of mTOR signaling: an emerging role for late endosome/lysosomal targeting. J Muscle Res Cell Motil. doi:10.1007/s10974-013-9367-4

Poggesi C, Ho CY (2014) Muscle dysfunction in hypertrophic cardiomyopathy: What is needed to move to translation? J Muscle Res Cell Motil. doi:10.1007/s10974-014-9374-0

van Eif VW, Bogaards SJ, van der Laarse WJ (2013) Intrinsic cardiac adrenergic (ICA) cell density and MAO-A activity in failing rat hearts. J Muscle Res Cell Motil. doi:10.1007/s10974-013-9373-6

Wong YY, Handoko ML, Mouchaers KT, de Man FS, VonkNoordegraaf A, van der Laarse WJ (2010) Reduced mechanical efficiency of rat papillary muscle related to degree of hypertrophy of cardiomyocytes. Am J Physiol Heart Circ Physiol 298:H1190-1197 